\title{
Geração de Imagens de Reflectância de um Ponto de Vista Geométrico
}

\author{
Reflectance Imaging of a Geometric Viewpoint
}

Marco Aurélio Oliveira da Silva ${ }^{1}$

Alexandre Curvelo de Andrade ${ }^{1}$

${ }^{1}$ AMS Kepler Engenharia de Sistemas

Av. Armando Lombardi, 800, sala 206, Barra da Tijuca - 22640-020 - Rio de Janeiro, RJ

Rua Alfredo Nogueira Ignácio Penido, 300, sala 58, 12246-000 - São José dos Campos, SP

$\{$ marco, curvelo\}@amskepler.com

\begin{abstract}
RESUMO - Este trabalho apresenta quatro métodos para gerar imagens de reflectância no topo da atmosfera e na superfície. Os métodos são classificados de acordo com faixa de varredura do sensor, estreita ou ampla. Os métodos para sensores com faixa de varredura estreita baseiam-se em LUTs, visto que a variabilidade do ângulo zenital solar dentro da cena pode ser ignorada. Para sensores com amplo campo de visada essa variabilidade é significativa e não deveria ser negligenciada. Os métodos para processar esse tipo de cena baseiam-se na determinação do fator de reflectância pixel-a-pixel. O modelo de transferência radiativa $6 \mathrm{~S}$ é usado para determinar o fator de reflectância bidirecional. A atmosfera é caracterizada pelos modelos disponibilizados pelo $6 \mathrm{~S}$ e por uma imagem de nebulosidade atmosférica. Os testes preliminares foram feitos com cenas LANDSAT-5 TM. As imagens produzidas são comparadas com as correspondentes de referência fornecidas pelo LEDAPS. Os resultados obtidos mostram concordância entre as imagens.
\end{abstract}

Palavras-chave: correção atmosférica, radiância, reflectância, ângulo zenital solar.

\begin{abstract}
This article presents four methods to produces TOA and surface reflectance factor images. The methods are sorted out in two categories in accordance to sensor swath width, namely narrow or wield swath width. For sensors with narrow swath width the methods are based on look-up tables, since the variability of solar zenith angle inside of the scene can be ignored. For sensors with wield swath width this one is significant and shouldn't be ignored. The methods to process scenes of this type are based on computation of reflectance factor pixel-a-pixel. The radiative transfer model $6 \mathrm{~S}$ is used to compute the bidirecional reflectance distribution function. The atmosphere is characterized by $6 \mathrm{~S}$ existent models and by atmospheric haziness image. The preliminaries tests have been done with scenes from LANDSAT-5 TM. The produced images by methods have been checked against the reference images provided by LEDAPS. The results have obtained show concordance between these images.
\end{abstract}

Keywords: atmospheric correction, radiance, reflectance, solar zenith angle.

\section{INTRODUÇÃO}

Conforme destaca Jensen (2009), a atmosfera exerce considerável influência nas imagens adquiridas por sensores em nível orbital. Este autor adverte sobre os vários fluxos de energia radiante provenientes de diferentes fontes que penetram no campo de visada e introduzem ruído ao processo de imageamento. Dependendo do tipo de estudo a ser desenvolvido a partir dessas imagens, a correção das distorções provocadas pela atmosfera pode influenciar ou não nos resultados (SONG et al., 2001). Os sensores em nível orbital coletam imagens em diferentes geometrias (geometria de visada e de iluminação). Tanto os ângulos azimutais e zenitais do sensor quanto da radiação solar incidente sofrem variações por conta destes efeitos, o que pode dificultar imensamente a diferenciação de imagens coletadas em momentos distintos. Por este motivo, é importante entender como os efeitos provocados pela atmosfera ao processo de imageamento modificam o comportamento de cada variável e qual a sua influência sobre as respostas espectrais dos alvos da superfície terrestre.

Algumas imagens possuem o campo de visada estreito. Seu ângulo zenital solar não varia muito dentro da faixa de varredura do sensor, logo, a área iluminada por um feixe de raios solares na superfície distorce pouco, de tal forma que os possíveis efeitos causados por esta degradação podem ser desconsiderados. Ferraz (2005), em seu estudo, por exemplo, aponta que em imagens com o campo de visada estreito, a alteração dos ângulos de elevação solar não influenciam significativamente os fatores de reflectância das bandas 03 (red) e 04 (NIR) do TM/LANDSAT-5, assim como os valores de NDVI calculados a partir destes.

No entanto, sensores como o WFI a bordo do CBERS-1/2/2B, por exemplo, que possuem campo de visada amplo, o mesmo feixe de raios solares varia consideravelmente. Nesses casos, deve-se considerar a variação do ângulo zenital solar como um componente importante. 
Neste artigo são apresentadas metodologias para a geração de imagens de reflectância no topo da atmosfera e na superfície. De acordo com a largura da faixa imageada verifica-se a necessidade ou não de considerar a variabilidade do ângulo zenital solar.

\section{CALIBRAÇÃO ABSOLUTA DE SENSORES ORBITAIS}

A calibração absoluta de um sensor orbital relaciona números digitais com medidas de radiância provenientes de objetos de referências no solo com caracterização espectral bem definida. Nesse procedimento são obtidos os parâmetros que definem uma relação linear entre número digital (ND) e radiância.

A determinação da radiância é um passo fundamental na conversão de dados de imagem em medidas de reflectância. Os valores calibrados de radiância mínimo e máximo que um sensor é capaz de registrar são fornecidos pelos responsáveis pelo satélite. A partir desses parâmetros, a conversão de ND em radiância é resolvida a partir de uma interpolação linear, conforme mostra a Eq. 1 .

$$
L_{\lambda}=\left(\frac{L M A X_{\lambda}-L M I N_{\lambda}}{N D_{\max }-N D_{\text {min }}}\right) \cdot\left(N D-N D_{\text {min }}\right)+L M I N_{\lambda}
$$

no qual,

- L $\lambda$ é a radiância espectral aparente $(\mathrm{W} /(\mathrm{m} 2 . \mathrm{sr} . \mu \mathrm{m}))$;

- LMAX入 é a radiância espectral máxima que o sensor é capaz de registrar $(\mathrm{W} /(\mathrm{m} 2 . \mathrm{sr} . \mu \mathrm{m}))$;

- LMIN $\lambda$ é a radiância espectral mínima que o sensor é capaz de registrar $(\mathrm{W} /(\mathrm{m} 2 . \mathrm{sr} . \mu \mathrm{m}))$;

- NDmax é o número digital correspondente a LMAX $\lambda$; e

- NDmin é o número digital correspondente a LMIN $\lambda$.

\section{REFLECTÂNCIA NO TOPO DA ATMOSFERA}

Segundo Ponzoni e Shimabukuro (2007), reflectância é a razão entre a radiação refletida pela radiação incidente, expressa através dos chamados Fatores de Reflectância ( $\rho$ ). Estes, por sua vez, podem ser bidirecionais, pois, frequentemente, existem duas geometrias envolvidas: 1. geometria de iluminação ou de incidência, formada pelos ângulos zenital e azimutal da fonte de iluminação e; 2 . a geometria de visada, formada pelos ângulos zenital e azimutal do sensor. Vale ressaltar que os valores de radiância obtidos através da Eq. 1 referem-se a sensores orbitais e, portanto, não representam exclusivamente o brilho do objeto observado pelo sensor, mas, da mistura de sinal da superfície com outros, provenientes dos efeitos provocados por sua passagem através da atmosfera. Logo, a reflectância calculada com base nesses valores é denominada Aparente, por tratar-se da reflectância calculada a partir do Topo da Atmosfera.
Como mostra a Eq. 2, descrita em Chander et al. (2009), os valores de reflectância aparente são obtidos a partir dos valores de radiância calculados pela Eq. 1 .

$$
\rho_{\lambda}=\frac{\pi \cdot L_{\lambda} \cdot d^{2}}{E S U N_{\lambda} \cdot \cos (\theta)}
$$

sendo que,

- $\rho \lambda$ é a reflectância aparente ou de topo da atmosfera (adimensional);

- L $\lambda$ é a radiância bidirecional aparente $(\mathrm{W} /(\mathrm{m} 2 . \mathrm{sr} . \mu \mathrm{m}))$;

- d é a distância Sol-Terra em unidades astronômicas:

- ESUN $\lambda$ é a irradiância solar exoatmosférica média no topo da atmosfera $(\mathrm{W} /(\mathrm{m} 2 . \mu \mathrm{m}))$; e

- $\theta$ é o ângulo zenital solar (rad).

O cosseno do ângulo zenital solar é calculado como mostra a Eq. 3.

$$
\cos (\theta)=\operatorname{sen}(\varphi) \cdot \operatorname{sen}(\delta)+\cos (\varphi) \cdot \cos (\delta) \cdot \cos (h)
$$

sendo, $\varphi$ a latitude, h o ângulo horário e $\delta$ a declinação solar (Eq. 4).

$$
\delta=23,45 \cdot \operatorname{sen}\left[\frac{360}{365}\right] \cdot(284+n)
$$

Na Eq. 4 n é o dia do ano para a data em questão.

\subsection{Influência do Ângulo Zenital Solar nas Medidas de Reflectância Aparente}

A Fig. 1a apresenta a variação do ângulo zenital solar dentro de uma região. A ideia é verificar o quanto essa variação influência nas medidas de reflectância aparente. Para isso foram definidas duas quadrículas de $8^{\circ}$ x $8^{\circ}$ centradas nas coordenadas $0^{\circ} \mathrm{S}$ e $52^{\circ} \mathrm{W}$ e $31^{\circ} \mathrm{S}$ e $52^{\circ} \mathrm{W}$, respectivamente.

Como pode ser visto nas Figs. 1a e 1c, a variação do ângulo zenital solar aumenta a medida que os pontos se afastam do centro. Isso reflete diretamente na reflectância aparente calculada, conforme mostram as Figs. 1b e 1d. As reflectâncias foram calculadas considerando uma medida constante de radiância que, de acordo com a variação de $\theta$, resultam em valores diferentes. O ideal é que alvos iguais dentro da cena apresentem o mesmo valor de reflectância. A diferença das medidas extremas de reflectância indica uma variação máxima em torno de $10 \%$ para a região testada numa data específica.

Pela Fig. 1 verifica-se que a consideração ou não da variação do ângulo zenital solar está intimamente ligada com a largura da faixa de varredura do sensor. No caso de cenas Landsat-5 TM em que a faixa de varredura é de 185 $\mathrm{km}$ essa variação pode ser desconsiderada. Já para o caso de cenas CBERS-2B WFI com faixa de varredura de 890 $\mathrm{km}$ essa variação passa a ser significativa e deveria ser considerada nas determinações dos fatores de reflectância. 

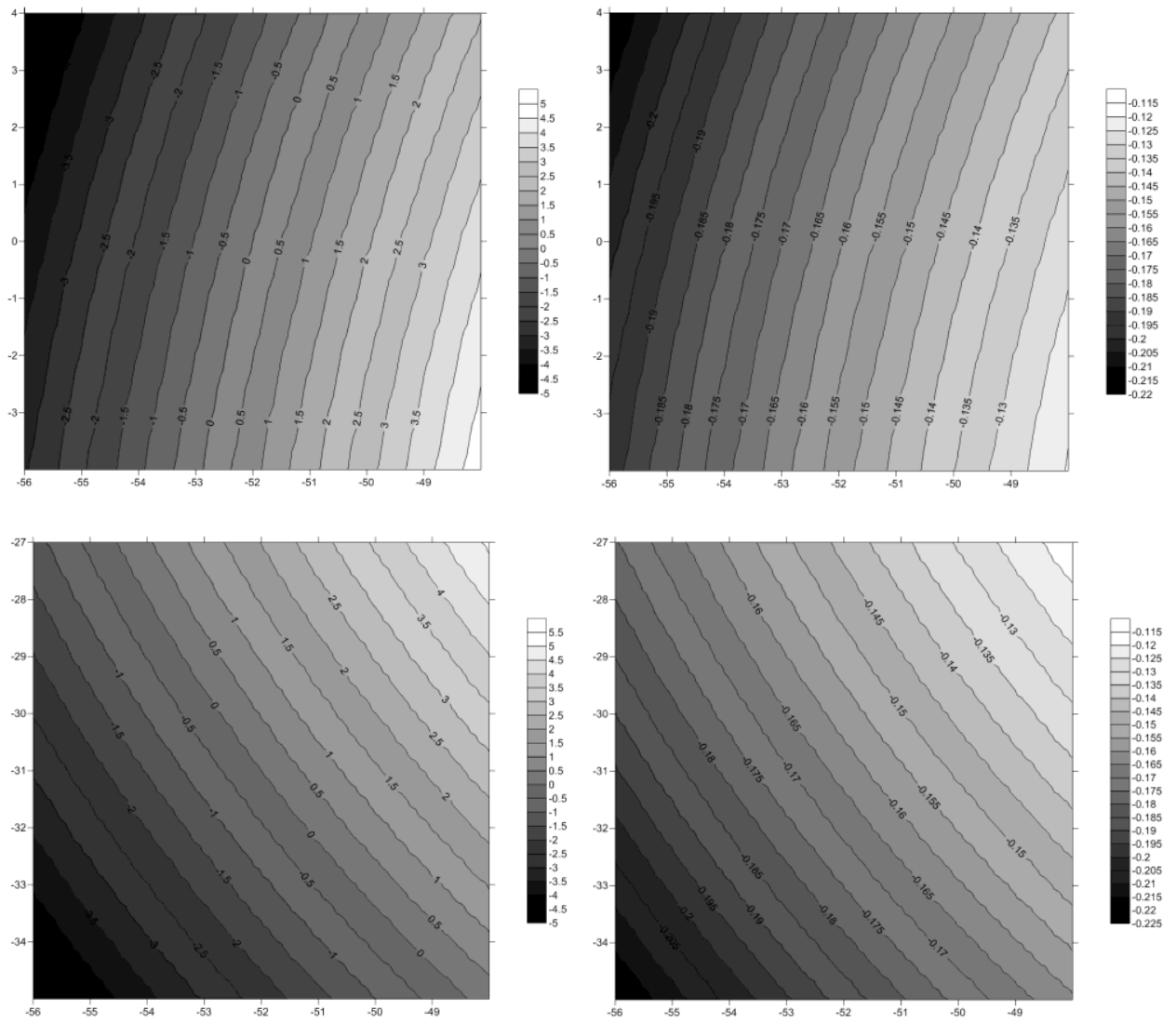

Figura 1 - Influência do Ângulo Zenital Solar na Reflectância Aparente. (a) Variação do Ângulo Zenital Solar na Quadrícula $8^{\circ}$ x $8^{\circ}$ centrada em $0^{\circ} \mathrm{S}$ e $52^{\circ} \mathrm{W}$; (b) Variação da Reflectância Aparente na Quadrícula $8^{\circ} \times 8^{\circ}$ centrada em $0^{\circ} \mathrm{S}$ e $52^{\circ} \mathrm{W}$; (c) Variação do Ângulo Zenital Solar na Quadrícula $8^{\circ}$ x $8^{\circ}$ centrada em $31^{\circ} \mathrm{S}$ e $52^{\circ} \mathrm{W}$; e (d) Variação da Reflectância Aparente na Quadrícula $8^{\circ}$ x $8^{\circ}$ centrada em $31^{\circ} \mathrm{S}$ e $52^{\circ} \mathrm{W}$.

\subsection{Geração de Imagens de Reflectância no Topo da Atmosfera}

\subsubsection{Sensores com campo de visada estreito}

Conforme foi mostrado anteriormente, a variação do ângulo zenital solar para sensores com visada estreita, tal como o Landsat-5 TM, é insignificante na determinação da reflectância. Dessa forma, uma única medida de ângulo zenital solar é suficiente para o processamento da cena inteira.

O algoritmo baseia-se no uso de look-up table (LUT). Uma única LUT é usada para o processamento da imagem original. A partir das equações 1 e 2 a LUT é construída convertendo-se números digitais em radiância aparente e, desta em reflectância aparente. O processamento é feito pixel-a-pixel, mapeando-se diretamente ND em reflectância aparente através da LUT.

\subsubsection{Sensores com amplo campo de visada}

Diferente do processamento para cenas com a faixa imageada estreita, aqui a variação do ângulo zenital solar deve ser considerada.
A solução apresentada anteriormente modifica-se um pouco nesse caso. Visto que o ângulo zenital solar deve ser calculado para todos os pixels da imagem, a criação de uma LUT de reflectância aparente é impossível. Cria-se, então, uma LUT intermediária de radiância aparente a partir da Eq. 1. O processamento é feito determinando-se a radiância através da LUT de radiância e calculando-se a reflectância aparente a partir da Eq. 2.

\section{REFLECTÂNCIA DE SUPERFÍCIE}

A refletância aparente é a razão entre a radiação refletida e a radiação incidente de um objeto localizado na superfície. Entretanto, ela é calculada a partir do topo da atmosfera, desconsiderando os possíveis efeitos provocados pelos constituintes atmosféricos nos fluxos radiantes incidentes e refletidos (Fig. 2). Dessa forma, os valores obtidos na Eq. 2 ainda não representam os valores físicos de reflectância de um objeto na superfície terrestre. A transformação dos fatores de reflectância aparente em fatores de reflectância de superfície, somente é possível após a eliminação ou mitigação dos efeitos provocados pela atmosfera. 


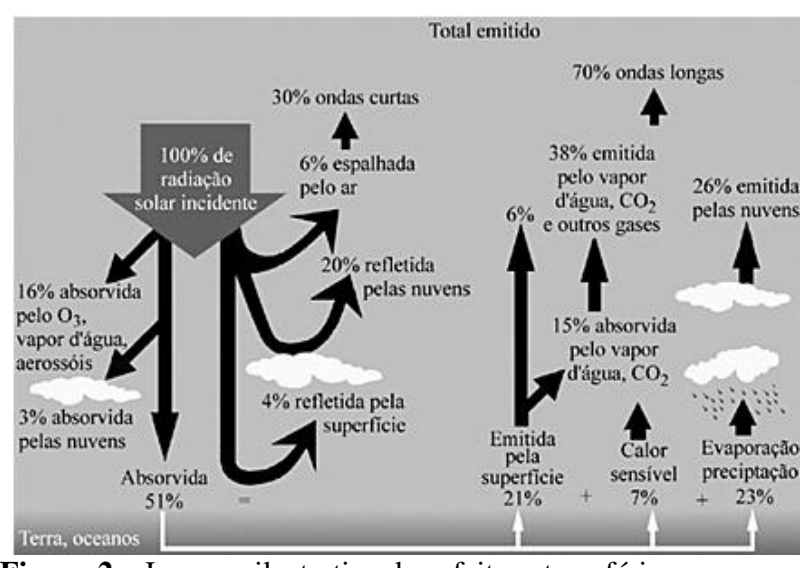

Figura 2 - Imagem ilustrativa dos efeitos atmosféricos.

Fonte: Martins et al. (2004).

\section{$4.16 S$}

O 6S (Second Simullation of a Satellite Signal in the Solar Spectrum) consiste num modelo de transferência radiativa desenvolvido para a correção atmosférica de imagens MODIS (Moderate Resolution Imaging Spectroradiometer) (Vermote, 1997b). Como se sabe, a radiação solar que atinge a superfície sofre vários efeitos de interação com a atmosfera que é constituída por gases e partículas de aerossol. Um modelo de transferência radiativa descreve essas interações matematicamente.

O 6S permite definir as geometrias de visada e de iluminação da cena através dos ângulos zenital e azimutal do sensor e do sol, caracterizar a atmosfera através de modelos atmosféricos e de aerossóis pré-definidos e através de perfis verticais de distribuição e da coluna total integrada de gases atmosféricos fornecidos pelo usuário. A quantidade de aerossóis é informada pela medida de visibilidade horizontal ou pela profundidade óptica espectral (Aerosol Optical Thickness (AOT)). Além disso, permite definir a altura do alvo, o sensor a bordo do satélite e a banda espectral.

Para utilizar o 6S na determinação dos coeficientes de correção atmosférica é necessário à definição de um arquivo de entrada com os parâmetros citados anteriormente. Um exemplo desse tipo de arquivo pode ser gerado através de uma ferramenta disponibilizada na própria página do $6 \mathrm{~S}$ (http://6s.ltdri.org). Para entender melhor seu formato é recomendado que o fonte main.f seja consultado.

\subsection{Geração de Imagens de Reflectância de Superfície}

Os algoritmos para a geração de imagens de reflectância de superfície têm como base a metodologia adotada pelo LEDAPS (Landsat Ecosystem Disturbance Adaptive Processing System). Tal metodologia foi adaptada para o processamento de cenas com amplo campo de visada. O 6S é usado na determinação do BRDF (Bidirecional Reflectance Distribution Function) para uma determinada posição geográfica num dado instante de tempo.

\subsubsection{Sensores com campo de visada estreito}

A geração de imagens de reflectância de superfície para cenas de sensores com campo de visada estreito também se baseia no uso de LUTs. O algoritmo pressupõe a existência de uma imagem de nebulosidade atmosférica. Nessa imagem tem-se uma medida de AOT associada a cada um dos pixels (Fig. 3).

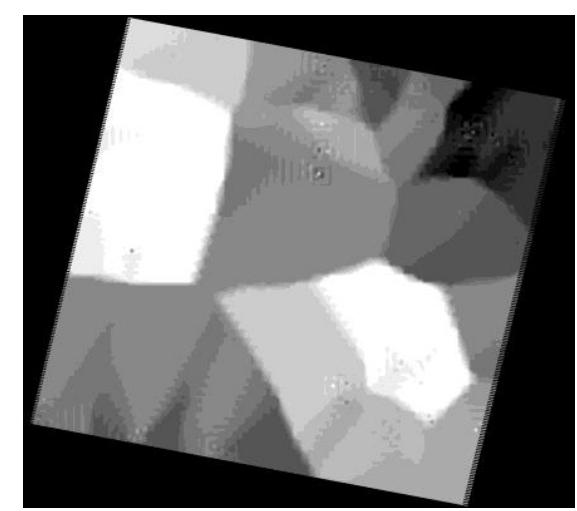

Figura 3 - Exemplo de uma Imagem de Nebulosidade Atmosférica.

São criadas várias LUTs de reflectância de superfície para diferentes medidas de AOT. Os valores devem ser selecionados de modo a melhor descrever a variabilidade de AOT dentro da cena. Um exemplo desse processo é a criação de duas LUTs considerando os valores máximo e mínimo de AOT.

Na construção de cada LUT é necessário caracterizar a atmosfera. Essa caracterização é feita através da definição dos parâmetros do modelo atmosférico (vapor d'água $\left(\mathrm{H}_{2} \mathrm{O}\right)$ e ozônio $\left.\left(\mathrm{O}^{3}\right)\right)$, dos parâmetros do modelo de aerossol (volumetria percentual da malha de aerossóis: poeira (dust), fuligem (soot), aerossóis oceânicos (oceanic) e partículas solúveis em água (water soluble)) e da nebulosidade da atmosfera. Juntamente a esses parâmetros têm-se aqueles que definem a geometria de visada e de iluminação da cena. Tais informações são processadas pelo $6 \mathrm{~S}$, no qual se têm como retorno três coeficientes de correção atmosférica $\left(\mathrm{x}_{\mathrm{a}}, \mathrm{x}_{\mathrm{b}}\right.$ e $\left.\mathrm{x}_{\mathrm{c}}\right)$. Como mostram as equações 5 e 6 , esses coeficientes relacionam reflectância de solo $\left(\rho s_{\lambda}\right)$ com radiância aparente.

$$
\begin{aligned}
& y=x_{a} \cdot L_{\lambda}-x_{b} \\
& \rho s_{\lambda}=\frac{y}{1+x_{c} \cdot y}
\end{aligned}
$$

A partir desse ponto fica claro a construção das LUTs. Para cada ND da imagem original determina-se a reflectância de solo correspondente através das equações 1 e 6.

Com as LUTs construídas para diferentes valores de AOT (Fig. 4a), o processamento de um i-ésimo pixel da imagem original inicia com a identificação do seu valor de AOT na imagem de nebulosidade atmosférica. Nesse ponto para cada pixel têm-se associado um ND (Fig. 4b) e 
uma medida de AOT (Fig. 4c). O próximo passo consiste na determinação da reflectância de superfície através das LUTs. As duas LUTs com AOT mais próximos ao do pixel são identificadas (Fig. 4d). Através dessas e do ND do pixel obtêm-se duas medidas de reflectância de superfície. Essas medidas são interpoladas, de acordo com o AOT do pixel em processamento, a fim de se obter sua medida de reflectância de superfície (Fig. 4e).

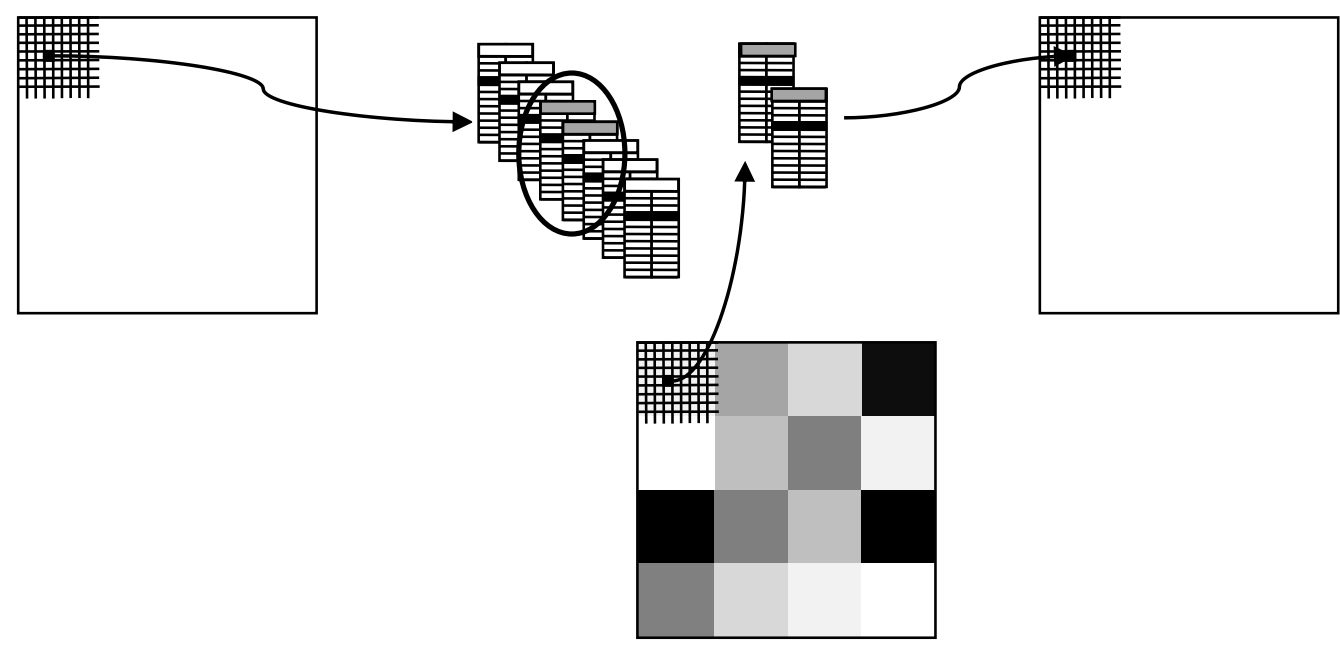

Figura 4 - Geração de Imagem de Reflectância de Superfície para Imagens com a Faixa de Varredura Estreita. (a) LUTs de Reflectância de Superfície para um Intervalo de AOT; (b) Imagem Original; (c) Identificação do valor de AOT do pixel sendo processado; (d) Duas LUTs de Reflectância de Superfície mais Próximas do AOT do Pixel em Processamento; e (e) Pixel de Reflectância de Superfície Obtido por Interpolação Linear.

\subsubsection{Sensores com amplo campo de visada}

Conforme foi dito anteriormente, para sensores com amplo campo de visada a variação do ângulo zenital dentro da cena passa ser significativa na determinação das medidas de reflectância aparente. Com base nisso, a determinação da reflectância aparente é feita pixel-a-pixel a partir da Eq. 2. O mesmo princípio poderia ser seguido aqui. Porém, o custo computacional torna esse processamento inviável para determinação da reflectância de superfície pixel-a-pixel. Para se ter idéia, o tempo consumido pelo $6 \mathrm{~S}$ na determinação de uma medida de reflectância de superfície é de aproximadamente 3 segundos. Considerando uma cena de 1000 x 1000 pixels o tempo de processamento seria de 833 horas. Dessa forma, simplificações são necessárias.

Uma simplificação que pode ser feita é a definição de uma grade amostral sobre a imagem original (Fig. 5a). Para cada nó da grade amostral são definidas as LUTs de reflectância de superfície para um intervalo de AOT. A Fig. 5b apresenta um exemplo de uma célula da grade amostral. Verifique que as LUTs para o intervalo de AOT são calculadas para cada um dos quatro cantos da célula. Perceba que as LUTs são criadas da mesma forma descrita na seção anterior. O problema de determinação da reflectância de superfície para imagens com amplo campo de visada se resume então, a $\mathrm{N}$ problemas de determinação de reflectância de superfície para imagens com campo de visada estreito.

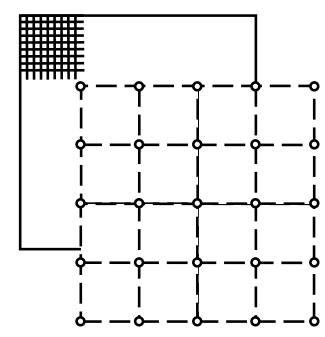

(a)

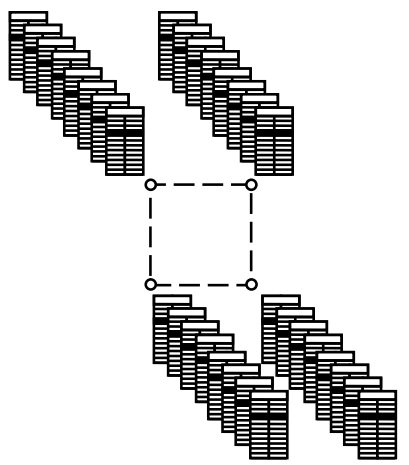

(b)

Figura 5 - Grade Amostral. (a) Definição da Grade Amostral sobre a Imagem Original; e (b) Determinação das LUTs de Reflectância de Superfície para os Nós da Grade Amostral.

Para cada pixel da imagem original (Fig. 6a) identifica-se a célula da grade amostral que o contêm (Fig. 6b). Na sequência, identifica-se o valor de AOT a partir da imagem de nebulosidade atmosférica (Fig. 6c). Têm-se então a medida de AOT e o ND do pixel sendo processado. Com esses valores determinam-se as medidas de reflectância de superfície para cada um dos cantos da célula através das LUTs (Fig. 6d). Como mostra a Fig. 6e a reflectância de superfície do pixel sendo processado é calculada interpolando-se bilinearmente as reflectâncias dos cantos. 


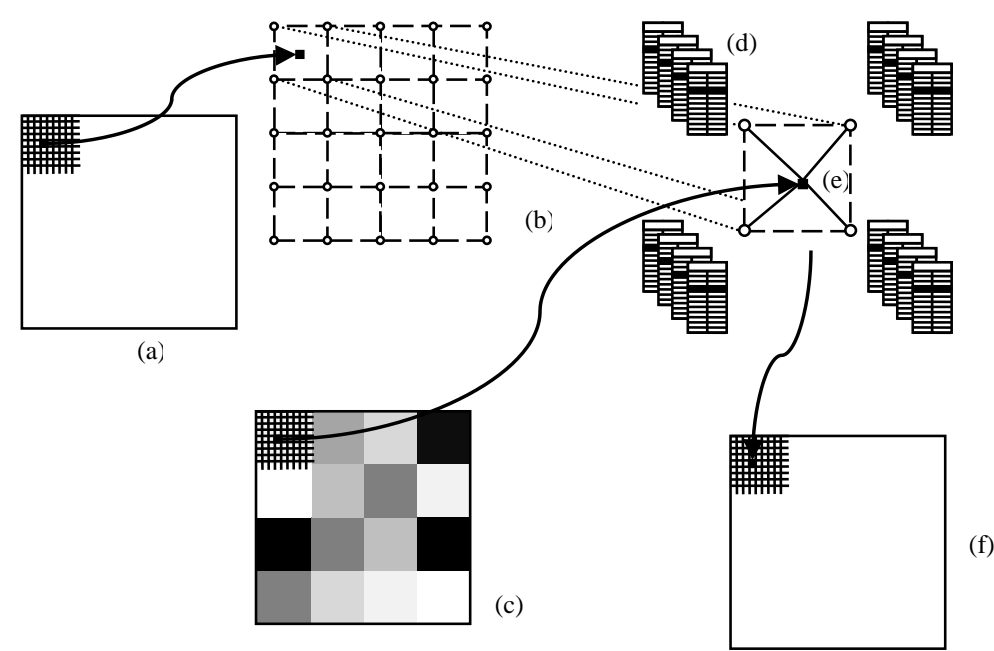

Figura 6 - Geração de Imagem de Reflectância de Superfície para Imagens de Sensores com Ampla Faixa de Varredura. (a) Imagem Original; (b) Identificação da Célula que Contem o Pixel Sendo Processado; (c) Identificação do valor de AOT do pixel sendo Processado; (d) Determinação da Reflectância de Superfície para Cada um dos Cantos da Célula a Partir das LUTs; (e) Interpolação Bilinear da Reflectância de Superfície do Pixel sendo Processado; e (f) Imagem de Reflectância de Superfície.

\section{RESULTADOS E ANÁLISE}

Os testes iniciais estão sendo conduzidos a partir de cenas Landsatt-5 TM. Para cada uma das cenas testadas foram obtidas, junto ao LEDAPS, as imagens de nebulosidade atmosférica, as de reflectância aparente e as de superfície. Foram usadas as cenas de path/row 177/033, 177/034 e 178/034 (Fig. 7). Essas três cenas encontram-se na Turquia. A cena 177/033 é do dia 06/09/2011 e tem centro nas coordenadas 38 $533^{\prime} 31^{\prime \prime} \mathrm{N}$ e $32^{\circ} 47^{\prime} 37$ "E. Essa cena cobre o lago salgado Tuz Golu, o qual é amplamente usado para propósitos de calibração radiométrica absoluta. A cena 177/034 também é do dia 06/09/2011 e tem centro nas coordenadas $37^{\circ} 27^{\prime} 57^{\prime \prime} \mathrm{N}$ e $32^{\circ} 21^{\prime} 54$ "E. Por fim, a cena 178/034 é do dia 29/09/2011 e tem centro nas coordenadas $37^{\circ} 28^{\prime} 00^{\prime \prime} \mathrm{N}$ e $30^{\circ} 50^{\prime} 18^{\prime \prime} \mathrm{E}$.

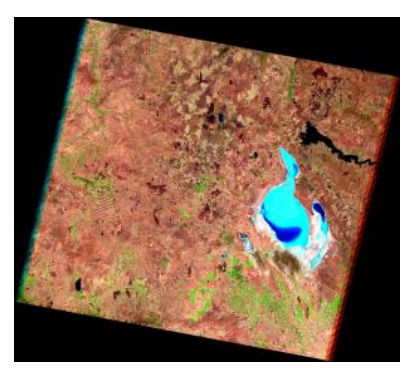

(a)

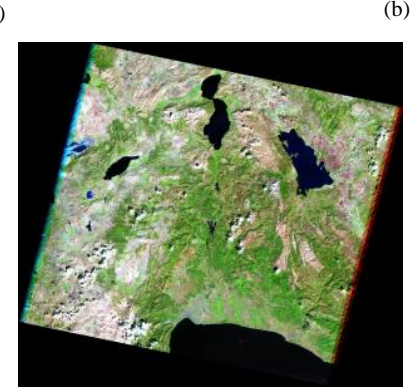

(c)

Figura 7 - Cenas usadas nos experimentos. (a) Landsat-5 TM 177/033; (b) Landsat-5 TM 177/034; e (c) Landsat-5 TM $178 / 034$.
Para realização dos testes o algoritmo foi configurado para gerar duas LUTs de reflectância de superfície a partir das ocorrências mínima e máxima de AOT.

A caracterização atmosférica foi feita a partir dos modelos pré-definidos do 6S. Foi adotado o modelo atmosférico mid-latitude summer e o modelo de aerossol continental. Os parâmetros de cada modelo são apresentados nas Tabs. 1 e 2.

Tabela 1 - Parâmetros do Modelo Atmosférico.

\begin{tabular}{lll}
\hline Modelo Atmosférico & \multicolumn{2}{c}{ Parâmetros } \\
\hline & $\mathrm{H}_{2} \mathrm{O}(\mathrm{g} / \mathrm{cm} 2)$ & $\mathrm{O}^{3}(\mathrm{~cm}-\mathrm{atm})$ \\
Mid-latitude Summer & 2,93 & 0,319 \\
\hline
\end{tabular}

Tabela 2 - Parâmetros do Modelo de Aerossol.

\begin{tabular}{lcccc}
\hline Modelo de Aerossol & \multicolumn{3}{c}{ Parâmetros } & \\
\hline & $\begin{array}{c}\text { Poeira } \\
(\%)\end{array}$ & $\begin{array}{c}\text { Partículas } \\
\text { Solúveis } \\
\text { em Água } \\
(\%)\end{array}$ & $\begin{array}{c}\text { Aerossóis } \\
\text { Oceânicos } \\
(\%)\end{array}$ & $\begin{array}{c}\text { Fuligem } \\
(\%)\end{array}$ \\
\hline Continental & 0,70 & 0,29 & 0,00 & 0,01 \\
\hline
\end{tabular}

Os resultados foram analisados comparando-se pixel-a-pixel as imagens geradas pelo algoritmo com as correspondentes produzidas pelo LEDAPS. Essa comparação resulta numa imagem diferença, sendo as estatísticas dessa imagem analisadas.

As tabelas a seguir apresentam as estatísticas das imagens de reflectância geradas pelo algoritmo (preenchimento branco), da imagem de reflectância produzida pelo LEDAPS (preenchimento cinza claro), bem como da imagem diferença (preenchimento cinza escuro).

$\mathrm{Na}$ Tab. 3 são apresentados os resultados obtidos para a cena $177 / 033$. Analisando-se essa tabela pode ser verificado que a diferença média entre as bandas processadas pelo algoritmo e pelo LEDAPS não foi 
superior a 3\%. A maior aproximação ocorreu na banda 1 . A diferença média nessa banda ficou em $0,24 \%$. O pior resultado ocorreu na banda 4 , em que a diferença média ficou em $2,5 \%$.

Tabela 3 - Estatísticas das Imagens de Reflectância de Superfície e da Imagem Diferença da Cena Landsat-5 TM 177/033.

\begin{tabular}{cllll}
\hline Banda & Máximo & Mínimo & Média & $\begin{array}{c}\text { Desvio } \\
\text { Padrão }\end{array}$ \\
\hline \multirow{2}{*}{1} & $-0,0877$ & 0,4068 & 0,1179 & 0,0691 \\
& $-0,1348$ & 0,4681 & 0,1061 & 0,0531 \\
& $-0,0631$ & 0,0420 & 0,0024 & 0,0085 \\
\hline \multirow{2}{*}{2} & $-0,0407$ & 0,8570 & 0,1778 & 0,0864 \\
& $-0,0830$ & 0,9130 & 0,1921 & 0,1029 \\
& $-0,1216$ & 0,0327 & $-0,0143$ & 0,0171 \\
\hline \multirow{2}{*}{3} & $-0,0193$ & 0,7278 & 0,2191 & 0,0924 \\
& $-0,0499$ & 0,8234 & 0,2361 & 0,1070 \\
& $-0,1054$ & 0,0258 & $-0,0170$ & 0,0154 \\
\hline \multirow{2}{*}{4} & 0,0033 & 0,9110 & 0,3090 & 0,0894 \\
& $-0,0129$ & 0,9797 & 0,3340 & 0,1010 \\
& $-0,988$ & 0,0226 & $-0,0250$ & 0,0133 \\
\hline \multirow{2}{*}{5} & 0,0011 & 0,6709 & 0,3524 & 0,0951 \\
& $-0,0167$ & 0,6723 & 0,3458 & 0,0952 \\
& $-0,0111$ & 0,0325 & 0,0064 & 0,0048 \\
\hline \multirow{2}{*}{7} & 0,0023 & 1,0387 & 0,2750 & 0,0833 \\
& $-0,0132$ & 1,0005 & 0,2604 & 0,0805 \\
\hline & 0,0008 & 0,0870 & 0,01432 & 0,0048 \\
\hline
\end{tabular}

A Tab. 4 apresenta os resultados obtidos para a cena 177/034. Como pode ser visto nessa tabela, a diferença média entre as bandas também não foi superior a $3 \%$. Nessa cena, a maior aproximação ocorreu na banda 2 , sendo que a diferença média ficou em $0.01 \%$. A maior diferença ficou na banda 7 . Essa foi de 2,5\%.

Tabela 4 - Estatísticas das Imagens de Reflectância de Superfície e da Imagem Diferença da Cena Landsat-5 TM $177 / 034$

\begin{tabular}{cllll}
\hline Banda & Máximo & Mínimo & Média & $\begin{array}{l}\text { Desvio } \\
\text { Padrão }\end{array}$ \\
\hline \multirow{2}{*}{1} & $-0,0951$ & 0,4045 & 0,0790 & 0,0383 \\
& $-0,0737$ & 1,6000 & 0,0903 & 0,0359 \\
& $-1,1955$ & 0,0193 & $-0,0113$ & 0,0085 \\
\hline \multirow{2}{*}{2} & $-0,0452$ & 0,8553 & 0,1304 & 0,0528 \\
& $-0,0360$ & 1,6000 & 0,1377 & 0,0517 \\
& $-0,7447$ & 0,0030 & $-0,0073$ & 0,0018 \\
\hline \multirow{2}{*}{3} & $-0,0227$ & 0,7248 & 0,1612 & 0,0740 \\
& $-0,0171$ & 1,6000 & 0,1645 & 0,0728 \\
& $-0,8752$ & 0,0036 & $-0,0034$ & 0,0020 \\
\hline \multirow{2}{*}{4} & $-0,0105$ & 0,9067 & 0,2825 & 0,0812 \\
& $-0,0058$ & 1,6000 & 0,2824 & 0,0805 \\
& $-0,6933$ & 0,0032 & 0,0001 & 0,0017 \\
\hline \multirow{2}{*}{5} & 0,0007 & 0,6649 & 0,3147 & 0,1101 \\
& $-0,0004$ & 1,6000 & 0,2928 & 0,1050 \\
& $-0,9351$ & 0,0478 & 0,0219 & 0,0197 \\
\hline \multirow{2}{*}{7} & 0,0021 & 1,0293 & 0,2395 & 0,0961 \\
& 0,0014 & 1,6000 & 0,2144 & 0,0867 \\
& $-0,5707$ & 0,1081 & 0,0251 & 0,0113 \\
\hline
\end{tabular}

A Tab. 5 apresenta os resultados obtidos para a cena 178/034. Dessa tabela, verifica que a diferença média entre as bandas não foi superior a $2 \%$. A maior aproximação ocorreu na banda 4 com diferença média de $-0,4 \%$. A maior diferença de $1,69 \%$ ficou na banda 7 .

Tabela 5 - Estatísticas das Imagens de Reflectância de Superfície e da Imagem Diferença da Cena Landsat-5 TM $178 / 034$.

\begin{tabular}{cllll}
\hline Banda & Máximo & Mínimo & Média & $\begin{array}{l}\text { Desvio } \\
\text { Padrão }\end{array}$ \\
\hline \multirow{2}{*}{1} & $-0,0998$ & 0,4538 & 0,0699 & 0,0471 \\
& $-0,0967$ & 1,6000 & 0,0857 & 0,1171 \\
& $-1,1462$ & 0,0217 & $-0,0159$ & 0,0834 \\
\hline \multirow{2}{*}{2} & $-0,0474$ & 0,9470 & 0,1041 & 0,0643 \\
& $-0,0496$ & 1,6000 & 0,1105 & 0,0677 \\
& $-0,6530$ & 0,0051 & $-0,0063$ & 0,0106 \\
\hline \multirow{2}{*}{3} & $-0,0236$ & 0,8017 & 0,1186 & 0,0763 \\
& $-0,0250$ & 1,6000 & 0,1226 & 0,0872 \\
& $-0,7983$ & 0,0058 & $-0,0040$ & 0,0254 \\
\hline \multirow{2}{*}{4} & $-0,0110$ & 1,0003 & 0,2191 & 0,0968 \\
& $-0,0148$ & 1,6000 & 0,2224 & 0,1004 \\
& $-0,5997$ & 0,0038 & $-0,0034$ & 0,0122 \\
\hline \multirow{2}{*}{5} & 0,0009 & 0,7336 & 0,2369 & 0,1290 \\
& $-0,0002$ & 1,6000 & 0,2247 & 0,1364 \\
& $-0,8664$ & 0,0484 & 0,0122 & 0,0433 \\
\hline \multirow{2}{*}{7} & 0,0024 & 1,1379 & 0,1785 & 0,1089 \\
& 0,0015 & 1,6000 & 0,1616 & 0,0991 \\
& $-0,4621$ & 0,1129 & 0,0169 & 0,0112 \\
\hline
\end{tabular}

\section{CONCLUSÕES}

Neste documento foram apresentadas metodologias para a geração de imagens de reflectância aparente e de superfície. Foi mostrado que o ângulo zenital solar pode influenciar nas reflectâncias calculadas dependendo do campo de visada do sensor, estreito ou amplo. Os experimentos realizados mostraram concordância das imagens produzidas pelo algoritmo com as correspondentes produzidas pelo LEDAPS.

Novos testes devem ser conduzidos com cenas provenientes de outros sensores com campo de visada amplo. Pode-se verificar a viabilidade de usar produtos MOD 09 também para validar as imagens geradas através da análise de alvos isolados.

Uma melhoria importante nos algoritmos desenvolvidos seria a geração automática de imagens de nebulosidade atmosférica. Além disso, a caracterização da atmosfera pode ser feita automaticamente a partir de imagens MODIS.

\section{AGRADECIMENTOS}

Este trabalho foi desenvolvido como parte da execução de um contrato entre o INPE e a AMS Kepler celebrado segundo a Lei de Inovação Tecnológica. 


\section{REFERÊNCIAS BIBLIOGRÁFICAS}

CHANDER, G.; MARKHAM, B. L.; HELDER, D. L. Summary of current radiometric calibration coefficients for Landsat MSS, TM, ETM+, and EO-1 ALI sensors. Remote Sensing of Environment, v 113, n. 5, p. 893-903, 2009.

FERRAZ, P. Influência da geometria de iluminação e do ângulo de visada na resposta espectral da cultura do feijão (Phaseolus vulgaris L.). 2005. 76 p. (CDD635.652). Dissertação de Mestrado em Irrigação e Drenagem. Escola Superior de Agricultura Luiz de Queiroz, Universidade de São Paulo, Piracicaba. 2005.

JENSEN, J. R. Sensoriamento remoto do ambiente: uma perspectiva em recursos terrestres. São José dos Campos: Parêntese, 2009. 604 p.

MARTINS, F. R.; PEREIRA, E. B.; ECHER, M. P. S. Levantamento dos recursos de energia solar no Brasil com o emprego de satélite geoestacionário - o Projeto Swera. Revista Brasileira de Ensino de Física, v 26, n. 2, p. 145-159, 2004.

PONZONI, F. J.; SHIMABUKURO, Y. E. Sensoriamento Remoto no Estudo da Vegetação. São José dos Campos: Parêntese, 2007. 127 p.

SONG, C.; WOODCOCK, C. E.; SETO, K. C.; LENNEY, M. P.; MACOMBER, S. A. Classification and change detection using LANDSAT TM Data: When and how to correct atmospheric effects? Remote Sensing of Environment, v. 75, n. 2, p. 230-244, 2001.

VERMOTE, E. F.; TANRÉ, D.; DEUZÉ, J. L.; HERMAN, M.; MORCRETTE, J. J. Second simulation of the satellite signal in the solar spectrum, 6s: An overview. IEEE Transactions on Geoscience and Remote Sensing. V. 35, n. 3, p. 675-686. MAY1997. 